Original research

\title{
Molecular investigation of extended-spectrum $\beta$-lactamases (ESBLs) genes in the Salmonella isolates obtained from children with acute diarrhea
}

Mohsen Mohammadi ${ }^{1}$, Mohammad Hossein Tayefeh-Arbab², Zohre Baseri3, Mojtaba Taghizadeh Armaki4,5, Mohsen Karami4,5, Saman Alhooei6,7, Abazar Pournajaf 2,4,*

${ }^{1}$ Non-Communicable Pediatric Diseases Research Center, Health Research Institute, Babol University of Medical Sciences, Babol, Iran ${ }_{2}^{2}$ Department of Microbiology, Faculty of Medicine, Babol University of Medical Sciences, Babol, Iran 3 Department of Pathology and Laboratory Medicine, Shariati hospital, Tehran University of Medical Sciences, Tehran, Iran ${ }_{4}$ Infectious Diseases and Tropical Medicine Research Center, Babol University of Medical Sciences, Babol, Iran

5 Department of Mycology and Parasitology, Faculty of Medicine, Babol University of Medical Sciences, Babol, Iran

${ }^{6}$ Department of Internal Medicine, Faculty of Medicine, Babol University of Medical Sciences, Babol, Iran

${ }_{7}$ Clinical Research Development Unite of Rouhani Hospital, Babol University of Medical Sciences, Babol, Iran

\begin{abstract}
Salmonellosis is an important public health concern among children in worldwide. Extended-spectrum $\beta$-lactams (ESBLs) cause resistance to clinically important beta-lactams which are generally used to treat invasive Salmonella infections. Therefore, the aim of this study was to investigate the presence of $S H V, T E M$ and CTX-M genes in different strains of Salmonella isolated from children with acute diarrhea and to determine their resistance profile. In this cross-sectional study, 300 fecal samples were collected from children referred to the Amirkola Children's Hospital, Babol, Iran. Antibiotic susceptibility testing was done according to the CLSI guideline. ESBLs-producing strains were identified using double disk synergy test method on the Mueller-Hinton agar plates. Multiplex-PCR was performed using oligonucleotide specific primers to detect of $S H V$, TEM and $C T X-M$ genes. In total, $7 \%(\mathrm{n} ; 21 / 300)$ salmonella were isolated, which $61.9 \%, 28.6 \%$ and $9.5 \%$ were Salmonella typhimurium, Salmonella enteritidis and Salmonella typhi, respectively. The prevalence of the ESBL-producing isolates were $52.4 \%$. M-PCR results showed that $42.8 \%, 38.1 \%$ and $14.3 \%$ of isolates were carried $C T X$ $M, T E M$ and $S H V$ genes, respectively. Also, $18.2 \%$ of isolates harbored $C T X-M$, and TEM genes, simultaneously. The high rate of ESBLs-producing Salmonella strains in the pediatric patients is an alarm. It is also recommended that alternative drugs be used with less resistance, which requires further investigation.
\end{abstract}

Keywords: Beta-lactamase, Salmonella, Diarrhea, Pediatric, PCR

\footnotetext{
${ }^{*}$ Corresponding author:

Dr. Abazar Pournajaf, Ph.D

Department of Microbiology, Faculty of Medicine,

Babol University of Medical Sciences, Babol, Iran

Tel/Fax: +9811 32190181

Email: abazar_pournajaf@yahoo.com

http://orcid.org/0000-0002-6753-5953.
}

Received: July, 05, 2020
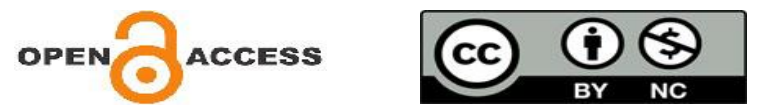

Accepted: August, 01, 2020 


\section{Introduction}

Salmonella, a member of the Enterobacteriaceae family, are Gram-negative bacilli and spore-free that most normally transmitted from animals to humans [1]. These intestinal organisms, which are often considered potential pathogens for humans, have more than 2,500 different serovars that are divided into three distinct species including, Salmonella typhi, Salmonella choleraesuis, and Salmonella enterica [2]. Salmonella entrica sub-species entrica serovar Enteritidis (briefly S. enteritidis) is the most important cause of Non-typhoidal Salmonellosis. This disease is one of the most important infectious diseases between humans and animals, which is mostly related to the consumption of meat, poultry, eggs and milk [3]. So this bacterium is a food-borne pathogen. Salmonellosis can develop into one of the forms of typhoid fever, septicemia, and gastrointestinal infections (gastroenteritis). Gastroenteritis is the most common form of salmonellosis, often associated with symptoms such as fever, abdominal cramps, and diarrhea. The most common serotypes involved in this form is S. typhimurium and S. enteritis [4]. The course of the disease lasts 4 to 7 days and many people recover without the need for antibiotics [5].

Today, one of the most important antimicrobials used in treatment of Salmonellosis is $\beta$-lactam agents. Unfortunately, for some reason, the addition of antibiotics to the diet of livestock, improper and arbitrary use of antibiotics, and lack of careful surveillance of drug administration have led to antibiotic-resistant strains [6]. The main problem in treating infections caused by these organisms is the emergence of multidrug resistance (MDR) strains, which often lead to prolonged hospitalization, increase mortality and mobility rates and increase the cost of treatment [7].

$\beta$-lactamases are a class of enzymes that hydrolysis the amide bond in the $\beta$-lactam ring of penicillins and cephalosporins. A common classification system is Ambler system which categorizes these enzyme into 4 dissimilar groups (A, B, C, D), based on the structure. Ambler type A (broadspectrum beta-lactamases (ESBL), C (Cephalosporinase), and D (oxacillinase) $\beta$ lactamases are categorized as a serine $\beta$-lactamases because they have ser amino acid at the enzyme's active site [8]. Ambler type B enzyme is classified as a metalloenzyme since the type need a divalent cations, usually zinc in active site to function. The spread of ESBLs is a global public health concern. ESBLs can develop resistance to antimicrobials such as 3 th and 4th generation cephalosporins and monobactams [9]. The most common type of ESBLs in clinical samples is SHV, whose coding gene is located on a transferable plasmid and is therefore easily distributed among bacterial strains [10]. SHV $\beta$-lactamases are inhibited by clavulanic acid but not by EDTA. The TEM- 1 was the first broad-spectrum $\beta$-lactamase, found not only in Enterobacteriaceae but also in Pseudomonas aeruginosa [11]. Among ESBLs, the CTX-M group has been described globally. The $C T X-M$ gene is located on a transferable plasmid and is effective on the cefotaxime (a 3th-generation cephalosporin). These enzymes have the ability to hydrolyze cephalosporins and are inhibited by clavulanic acid, sulbactam, and tazobactam. CTX-M types are widely spread around the world. Although Salmonella serotypes are a significant etiologic agents of diarrhea in various parts of the world, very few reports are obtainable about the incidence of ESBLs among them [12]. So, the aim of the current study is to detect ESBL-encoded genes (TEM, SHV and CTX-M) in the Salmonella isolated from children with acute diarrhea and determination of the resistance pattern of these strains.

\section{Materials and Methods}

2.1. Bacterial isolation

In this cross-sectional study, which was performed over a period of 9 months (from the February to October 2019), in total 300 stool samples were obtained from the children's suspected to Salmonellosis at the Amirkola Children's Hospital, Babol, Iran. In laboratory, each sample was enriched using culturing on the Selenite-F Broth (Mercury, Germany). After incubation on the $37^{\circ} \mathrm{C}$ for $24 \mathrm{~h}$, samples were cultured on the Eosin Methylene Blue (EMB) agar and Xylose Lysine Deoxycholate (XLD) agar (Merck, Germany) plates. Lactose negative colonies were identified by API $20 \mathrm{E}$ system (BioMerieux, France). All Salmonella spp. Isolates were serotyped with Salmonella specific $\mathrm{O}$ and $\mathrm{H}$ antisera using the slide agglutination test.

2.2. Antimicrobial susceptibility test

In according to the Standard Institute of Laboratory and Clinical Standards (CLSI), Antimicrobial susceptibility test was determined using agar disk diffusion method on the Mueller-Hinton agar (MHA) (Merck Co, Germany) for ceftazidime (30 
$\mu \mathrm{g}$ ), Aztreonam (30 $\mu \mathrm{g}$ ), Imipenem (10 $\mu \mathrm{g})$, Cefotaxime (30 $\mu \mathrm{g})$, Ofloxacin (5 $\mu \mathrm{g})$, Amikacin (30 $\mu \mathrm{g})$ and Tetracycline (30 $\mu \mathrm{g})$ (HiMedia, India) [13].

2.3. Double disk synergy test (DDST)

DDST was used for ESBL-producing isolates, cefotaxime $30 \mu \mathrm{g}$ and ceftazidime $30 \mu \mathrm{g}$ with and without clavulanic acid $10 \mu \mathrm{g}$ (Mast, UK). The 0.5 McFarland standard turbidity of isolates were spread on the Muller Hinton agar (Merck, Germany). DDST was done by comparing the inhibition zone of disks containing cefotaxime and ceftazidime with and without clavulanic acid. When zones were distended < $5 \mathrm{~mm}$ around the disk comprising clavulanic acid the strain were considered as ESBL positive. Klebsiella pneumoniae ATCC 7006039 was used as a positive control [14].

\subsection{Multiplex PCR}

Cellular DNA was extracted by the boiling way formerly defined previously [15]. The M-PCR was done using specific primers to identify $S H V$, TEM and $C T X-M$ genes (Table 1). The PCR reaction was performed at a volume of $25 \mu$ l. Each PCR reaction includes, $1.5 \mu \mathrm{L}$ of template DNA, $12.5 \mu \mathrm{L}$ of CinnaGen PCR Master Mix, 1.0 $\mu \mathrm{L}$ of each primer, and $9 \mu \mathrm{L}$ of ddH2O. The M-PCR reaction was performed for 33 cycles in an Eppendorf MasterCycle Gradient thermocycler (Eppendorf, Germany) with the fallowing program, initial denaturation at $94^{\circ} \mathrm{C}$ for 6 min, denaturation at $95^{\circ} \mathrm{C}$ for $45 \mathrm{~s}$, annealing at $57^{\circ} \mathrm{C}$ for $60 \mathrm{~s}$, extension at $72^{\circ} \mathrm{C}$ for $60 \mathrm{~s}$ and a final extension at $72^{\circ} \mathrm{C}$ for $7 \mathrm{~min}$. M-PCR amplicons were electrophoresed in a $1 \%$ agarose/0.5 × TBE $(45 \mathrm{mM}-$ Tris-borate, $1 \mathrm{mM}$-EDTA) gel stained with $0.1 \mu \mathrm{l} / \mathrm{ml}$ Gel Red ${ }^{\mathrm{TM}}$ (Biotium, USA), then photographed under an UV trans-illuminator. The 100-bp DNA ladder (CinnaGen, Iran) was used as a molecular size marker.

\section{Results}

The mean age of the patients was $13.6 \pm 1.2$ years (range from 4 to 14 years), which $45.3 \%$ (n; 163) were female and $45.6 \%(n ; 137)$ male. In total, $7 \%(n$; 21/300) salmonella species were collected, which $61.9 \%(n ; 13 / 21), 28.6 \%$ (n; 6/21) and 9.5\% (n; 2/21) were $S$. typhimurium, $S$. enteritidis and $S$. typhi, respectively. Antibiotic susceptibility test showed that all isolates were susceptible to imipenem (Table 2). Moreover, $42.8 \%$ ( $\mathrm{n}$; $9 / 21)$ of isolates were resistant to at least 3 different antibiotic classes and therefore considered MDR. Most MDR strains belonged to $S$. typhimurium serotype.
The results of DDST showed that $52.4 \%(n ; 11)$ of isolates had ESBL-positive phenotype. The results of M-PCR showed that $42.8 \%(\mathrm{n} ; 9), 38.1 \%(\mathrm{n} ; 8)$ and $14.3 \%(\mathrm{n} ; 3)$ of isolates were carried CTX-M, TEM and $S H V$ genes, respectively. Also, $18.2 \%(\mathrm{n} ; 2)$ of isolates carried genes $C T X-M$, and TEM simultaneously.

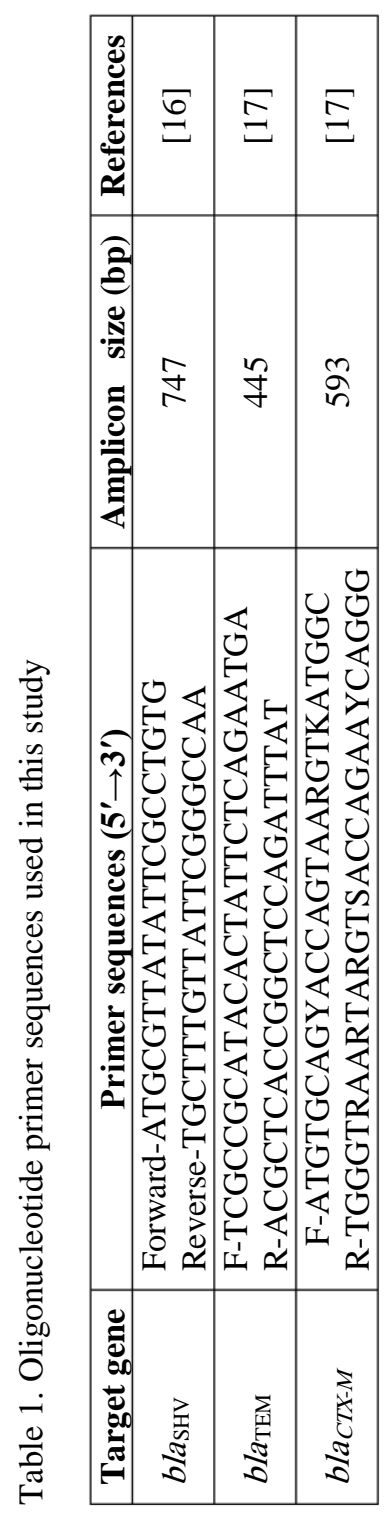


Table 2. Antibiotic susceptibility profile

\begin{tabular}{|l|c|c|c|c|c|c|c|c|c|}
\hline \multirow{2}{*}{ Antimicrobial agents } & \multicolumn{3}{|c|}{ S. typhimurium $\mathbf{n}=13)$} & \multicolumn{3}{c|}{ S. enteritidis $(\mathbf{n}=6)$} & \multicolumn{3}{c|}{ S. typhi $(\mathbf{n}=\mathbf{2})$} \\
\cline { 2 - 10 } & $\mathrm{S}$ & $\mathrm{I}$ & $\mathrm{R}$ & $\mathrm{S}$ & $\mathrm{I}$ & $\mathrm{R}$ & $\mathrm{S}$ & $\mathrm{I}$ & $\mathrm{R}$ \\
\hline Ceftazidime & $3(23.1)$ & $1(7.7)$ & $9(69.2)$ & $1(16.6)$ & 0 & $5(83.3)$ & 0 & 0 & $2(100)$ \\
\hline Aztreonam & $4(30.7)$ & $1(7.7)$ & $8(61.5)$ & $2(33.3)$ & 0 & $4(66.6)$ & 0 & 0 & $2(100)$ \\
\hline Imipenem & $13(100)$ & 0 & 0 & $6(100)$ & 0 & 0 & $2(100)$ & 0 & 0 \\
\hline Cefotaxime & $4(30.7)$ & 0 & $9(69.2)$ & $2(33.3)$ & 0 & $4(66.6)$ & 0 & 0 & $2(100)$ \\
\hline Ofloxacin & $8(61.5)$ & $1(7.7)$ & $4(30.7)$ & $4(66.6)$ & $1(16.6)$ & $1(16.6)$ & $1(50)$ & $1(50)$ & 0 \\
\hline Amikacin & $2(15.4)$ & $2(15.4)$ & $9(69.2)$ & $5(83.3)$ & 0 & $1(16.6)$ & 0 & 0 & $2(100)$ \\
\hline Tetracycline & $3(23.1)$ & 0 & $10(76.9)$ & $1(16.6)$ & 0 & $5(83.3)$ & 0 & 0 & $2(100)$ \\
\hline Ceftriaxone & $12(92.3)$ & 0 & $1(7.7)$ & $5(83.3)$ & 0 & $1(16.6)$ & $2(100)$ & 0 & 0 \\
\hline Ciprofloxacin & $12(92.3)$ & 0 & $1(7.7)$ & $6(100)$ & 0 & 0 & $2(100)$ & 0 & 0 \\
\hline
\end{tabular}

Results presented as No. (\%)

Abbreviations: S; susceptible; I: intermediate-resistant; R: resistant

\section{Discussion}

Diarrheal diseases caused by Salmonella are a worldwide health problem. Most people improve without specific treatment. Antibiotics are usually used only to treat cases suffering from severe infection. Patients should drink extra liquids as long as diarrhea lasts. In some cases, diarrhea may be so severe that the person needs to be hospitalized. Occasionally, infection may spread from the intestines to the bloodstream, and then to other parts of the body. In these cases, Salmonella can cause death unless the person is treated promptly with antibiotics. $\beta$ lactamases are extensively used in the treatment of salmonellosis [1]. In the present study, out of 300 stool samples, $7 \%$ Salmonella spp. were obtained, which is consistent with the study directed by Nodeh Farahani et al. [18]. This contradiction can be related to the number of samples and the year of study. In agreement with Soltan Dallal et al. [19], slide agglutination test results showed that $61.9 \%, 28.6 \%$ and $9.5 \%$ of isolates were belonged to the $S$. typhimurium, S. enteritidis and $S$. typhi serotypes, respectively. This variation may be resulted from distribution of different serotypes in various geographical areas.

Antimicrobial susceptibility test showed that all strains were susceptible to the imipenem, which was fully consistent with the study of Eshraghi et al. [20]. Also, $80.9 \%$ of isolates were resistant to tetracycline which was consistent with findings of Siourimè et al. [21]. In our study, with the exception of only one strain (S. enteritidis), all of them (20 isolates; 95.2\%) were sensitive to ciprofloxacin, which is almost consistent with the results of Eshraghi et al. [20]. The researchers showed that the favorable effect of quinolones in combination with cephalosporins. Spiliopoulou et al. showed that all isolates were sensitive to ceftriaxone and ciprofloxacin, which is consistent with the present study [22]. This agreement could be due to the lack of need for antibiotics in the treatment of salmonellosis and its self-limitation.

In the DDST test, $52.4 \%$ of isolates were ESBLs producing phenotype. Also, the prevalence of $C T X-M$, TEM and $S H V$ genes were $42.8 \%, 38.1 \%$, and $14.3 \%$, respectively. These data are inconsistent with the study of Tajbakhsh et al., since that all 174 Salmonella species were negative for TEM, CTX-M and $S H V$ genes [23]. These results could be due to the geographical distance and the presence of other resistance mechanisms such as, efflux pumps, chromosomal beta-lactamase and porin loss. Of $138 S$. enterica, 29.6\% (n; 40) ESBL-producing isolates were collected by Ranjbar et al. [24]. The frequency of CTX$M, T E M$ and $S H V$ genes was $12.3 \%(\mathrm{n} ; 17), 29.9 \%$ (n; 40) and $2.9 \%$ (n; 4), respectively, which was different with the present study. These results are important as an alarming in the development of resistance genes to the other susceptible strains and special attention to these strains. All 92 Salmonella spp. collected by Boisramé-Gastrin et al. were positive for TEM gene. So, $22.8 \%(n ; 21 / 92)$ and $5.4 \%(n ; 21 / 92)$ of isolates were harbored $S H V$ and $C T X-M$ genes, respectively [25]. The present study provided information on the frequency and antimicrobial susceptibility profile of Salmonella isolates in Iran. The high rates of ESBLs positive-Salmonella strains collected from pediatric 
cases is alarming and indicates a necessity to substitute the cephalosporins with an appropriate alternative.

Current limitations include the limited number of serotypes identified and resistance genes. It is also suggested that other resistance genes and other types of beta-lactamases should be identified.

Accurate diagnosis and rapid identification of ESBLs-producing Salmonella strains from pediatric patients is very important. Therefore, continuous monitoring of these resistances, especially at the endemic region, is an obvious thing that can prevent the spread of resistance to other strains and reduce the cost of treatment.

\section{Acknowledgments}

Aspecial note of thank goes to Amirkola Children's Hospital for providing patients specimen, as well as epidemiological and demographic data.

\section{Author Contributions}

All authors contributed similarly to this work, and confirm the final version of manuscripts.

\section{Conflict of Interests}

Authors declare there is no conflict of interest. Ethical declarations

All samples were obtained from patients as parts of routine sampling during their hospitalization period, so the regional ethical committee waived the need for informed consent.

\section{Financial Support}

Self-funding.

\section{References}

1. Gordon MA, Feasey NA, Graham SM. 45 - Nontyphoid Salmonella Disease. In: Magill AJ, Hill DR, Solomon T, Ryan ET, editors. Hunter's Tropical Medicine and Emerging Infectious Disease (Ninth Edition). London: W.B. Saunders; 2013. p. 462-7. 2. Gast, RK and Porter, RE., Jr. Salmonella Infections. In Diseases of Poultry. (eds D.E. Swayne, M. Boulianne, CM. Logue, LR. McDougald, V. Nair, DL. Suarez, S. Wit, T. Grimes, D. Johnson, M. Kromm, TY. Prajitno, I. Rubinoff and G. Zavala). 2020. p. 717-53. 3. Sevilla-Navarro S, Catalá-Gregori P, García C, Cortés V, Marin C. Salmonella Infantis and Salmonella Enteritidis specific bacteriophages isolated form poultry faeces as a complementary tool for cleaning and disinfection against Salmonella. Comp Immunol Microb. 2020; 68:101405.

4. Kadhim HM. Review of pathogenicity and virulence determinants in Salmonella. EurAsian J Biosci. 2020; 14(1):37781.

5. Lee CM, Lee MS, Yang TL, Lee KL, Yen TY, Lu CY, et al. Clinical features and risk factors associated with bacteremia of nontyphoidal salmonellosis in pediatric patients, 2010-2018. J Formos Med Assoc. 2020. [In press]. doi: 10.1016/j.jfma.2020.04.022.
6. Lingzhi L, Haojie G, Dan G, Hongmei M, Yang L, Mengdie J, et al. The role of two-component regulatory system in $\beta$-lactam antibiotics resistance. Microbiol Res. 2018; 215:126-9.

7. AL_Jubori SS, AL_Kadmy IM, JassimAl_Ani Z. Emergence of multidrug resistance (MDR) Acinetobacter baumannii isolated from Iraqi hospitals. Adv Environ Biol. 2016; 10(5):265-76.

8. Bush K, Jacoby GA. Updated functional classification of betalactamases. Antimicrob Agents Chemother. 2010; 54(3):969-76.

9. Bush K, Bradford PA. Epidemiology of $\beta$-Lactamase-Producing Pathogens. Clin Microbiol Rev. 2020; 33(2):eooo47-19.

10. Sawa T, Kooguchi K, Moriyama K. Molecular diversity of extended-spectrum $\beta$-lactamases and carbapenemases, and antimicrobial resistance. J Intensive Care. 2020; 8:13.

11. Kwok KO, Chan E, Chung PH, Tang A, Wei WI, Zhu C, et al. Prevalence and associated factors for carriage of Enterobacteriaceae producing ESBLs or carbapenemase and methicillin-resistant Staphylococcus aureus in Hong Kong community. J Infect. 2020; 81(2):242-7.

12. Kawamura K, Sugawara T, Matsuo N, Hayashi K, Norizuki C, Tamai K, et al. Spread of CTX-Type Extended-Spectrum $\beta$ Lactamase-Producing Escherichia coli Isolates of Epidemic Clone B2-O25-ST131 Among Dogs and Cats in Japan. Microb Drug Resist. 2017; 23(8):1059-66.

13. CLSI. Performance Standards for Antimicrobial Susceptibility Testing. 29th ed. CLSI supplement M10o. Wayne, PA: Clinical and Laboratory Standards Institute, 2019.

14. Gajdács M, Urbán E. Epidemiological Trends and Resistance Associated with Stenotrophomonas maltophilia Bacteremia: A 10Year Retrospective Cohort Study in a Tertiary-Care Hospital in Hungary. Diseases. 2019; 7(2):41.

15. Nobari S, Shahcheraghi F, Rahmati Ghezelgeh F, Valizadeh B. Molecular characterization of carbapenem-resistant strains of Klebsiella pneumoniae isolated from Iranian patients: first identification of blaKPC gene in Iran. Microb Drug Resist. 2014; 20(4):285-93.

16. Monstein HJ, Ostholm-Balkhed A, Nilsson MV, Nilsson M, Dornbusch K, Nilsson LE. Multiplex PCR amplification assay for the detection of blaSHV, blaTEM and blaCTX-M genes in Enterobacteriaceae. Apmis. 2007; 115(12):1400-8.

17. Ghasemi Y, Archin T, Kargar M, Mohkam M. A simple multiplex PCR for assessing prevalence of extended-spectrum $\beta$ lactamases producing Klebsiella pneumoniae in Intensive Care Units of a referral hospital in Shiraz, Iran. Asian Pac J Trop Med. 2013; 6(9):703-8.

18. Nodeh Farahani N, Masjedian Jazi F, Nikmanesh B, Asadolahi P, Sadeghi Kalani B, Amirmozafari N. Prevalence and Antibiotic Susceptibility Patterns of Salmonella and Shigella Species Isolated from Pediatric Diarrhea in Tehran. Arch Pediatr Infect Dis. 2018; 6(4):e57328.

19. Soltan Dallal MM, Rastegar Lari A, Sharifi Yazdi MK. [Pattern of serotyping and antibiotic resistance of Salmonella in children with diarrhea]. J Gorgan Uni Med Sci. 2014; 16(1): 100-105. [In Persian]

20. Eshraghi S, Dalall MMS, Fardsanei F, Salehi TZ, Ranjbar R, Nikmanesh B, et al. Salmonella enteritidis and antibiotic resistance patterns: A study on 1950 children with diarrhea. Tehran Univ Med J. 2010; 67(12):876-82.

21. Siourimè SN, Isidore BOJ, Oumar T, Nestor BIH, Yves $\mathrm{T}$, Nicolas B, et al. Serotyping and antimicrobial drug resistance of 


\section{Mohammadi et al.}

Salmonella isolated from lettuce and human diarrhea samples in Burkina Faso. Afr J Infect Dis. 2017; 11(2):24-30.

22. Spiliopoulou I, Zografou S, Goula A, Dimitracopoulos G, Christofidou M. Molecular epidemiology and antibiotic resistance patterns of Salmonella enterica from southwestern Greece. Chemotherapy. 2007; 53(6):392-6.

23. Tajbakhsh M, Avini MY, Khajeh JA, Alebouyeh M, Mojarad $\mathrm{EN}$, Zali MR. Increased-resistance phenotype resulted from elevated $\beta$-lactamase enzyme activity in Salmonella clinical isolates. J Isfahan Med Sch. 2012; 30(178).
24. Ranjbar R, Ardashiri M, Samadi S, Afshar D. Distribution of extended-spectrum $\beta$-lactamases (ESBLs) among Salmonella serogroups isolated from pediatric patients. Iran J Microbiol. 2018; 10(5):294-9.

25. Boisramé-Gastrin S, Tandé D, Münck MR, Gouriou S, Nordmann P, Naas T. Salmonella carriage in adopted children from Mali: 2001-08. J Antimicrob Chemother. 2011; 66(10):22716. 